\title{
Surgical and Survival Outcomes with Neoadjuvant Chemotherapy in Advanced Epithelial Ovarian Cancer: A Longitudinal Study in a Tertiary Cancer Center
}

\author{
Rekha B Raghavendrachar ${ }^{1}$, Rani A Bhat ${ }^{2}$, Vibhawari Dhakaria $^{3}$
}

\begin{abstract}
Aims: To evaluate surgical and survival outcomes following neoadjuvant chemotherapy followed by interval debulking surgery (IDS) in advanced epithelial ovarian cancer (AEOCs).

Materials and methods: Fifty patients who were diagnosed with stage III-IV ovarian cancer/primary peritoneal cancer/fallopian tube cancer were followed up following three cycles of neoadjuvant chemotherapy and debulking surgery followed by adjuvant chemotherapy in a comprehensive cancer center. This longitudinal study was conducted over four years from 2013-2017. Primary outcome measured was progression-free survival and secondary outcomes that included overall survival and effect of optimal and suboptimal surgeries on survival outcomes. In this study, we compared the difference in survival outcomes following various types of debulking (complete vs optimal vs suboptimal).

Results: Among 50 patients who underwent debulking, $78 \%, 8 \%$, and $14 \%$ underwent complete, optimal, and suboptimal debulking, respectively. The mean estimate of progression-free survival (PFS) at 24 months was $20.324 \pm 0.97$ months ( $95 \% \mathrm{Cl}$ ) and the overall survival was $21.234 \pm$ 0.788 months. There was a significant increase in PFS in those who had complete debulking ( $21.47 \pm 0.95$ months, $95 \% \mathrm{Cl} 19.6$ to 23.3 months) compared to those in optimal debulking (20.53 months) and suboptimal groups (10.3 months) (Log-rank Mantel-Cox $=12.01, p=0.001$ ). Seventy-eight percent had no postoperative complications while $16 \%$ had grade I complications. Grade II, IV, and V complications were $2 \%$ each. Conclusion: Neoadjuvant chemotherapy followed by IDS gives better chance of complete debulking and better survival outcomes with acceptable postoperative complications.

Keywords: Cytoreductive surgery, Epithelial ovarian cancer, Interval debulking surgery, Neoadjuvant chemotherapy, Survival outcomes. Journal of South Asian Federation of Obstetrics and Gynaecology (2020): 10.5005/jp-journals-10006-1751
\end{abstract}

\section{INTRODUCTION}

Epithelial ovarian cancer (EOC) is the leading cause of death in women with gynecological malignancy. According to GLOBOCAN 2018 data, there were an estimated 295,414 new cases of ovarian cancer worldwide in 2018 and 184,799 deaths. This amounts to $3.4 \%$ of all cancers in women and $4.4 \%$ of all cancer-related deaths in women. ${ }^{1}$ In India, there were 36,170 new cases of ovarian cancer in 2018 making it the third most common cancer in women with an incidence of 5.5 per 100,000 women. ${ }^{1}$ The National Cancer Registry Program (NCRP) in its report has shown that the age-adjusted incidence rates for ovarian cancer shows an increasing trend over the last 5 years, which is statistically significant. ${ }^{2}$

Majority of EOC present in advanced stage due to lack of symptoms and screening tools. Primary debulking surgery followed by adjuvant chemotherapy is the standard of care for ovarian cancer and complete cytoreduction is the most important predictor of survival. ${ }^{3}$ The presence of extensive peritoneal disease, upper abdominal disease, and visceral involvement makes optimal cytoreduction a difficult task to achieve and involves high rates of morbidity and mortality. Therefore as an alternative to the standard treatment, neoadjuvant chemotherapy (NACT) followed by interval debulking surgery (IDS) was evaluated in several studies and was found to be noninferior to primary debulking surgery (PDS). ${ }^{4}$

Neoadjuvant chemotherapy leads to improved quality of life by reducing the disease burden to some extent and resolution of ascites and/or pleural effusion. But, NACT has been questioned with regards to platinum resistance developing in the patient and also the significant side effects that can postpone the surgical
${ }^{1}$ Department of Gynecologic Oncology, Sri Shankara Cancer Hospital and Research Centre, Bengaluru, Karnataka, India

2,3 Department of Gynecologic Oncology, HCG Enterprises, Bengaluru, Karnataka, India

Corresponding Author: Rekha B Raghavendrachar, Department of Gynecologic Oncology, Sri Shankara Cancer Hospital and Research Centre, Bengaluru, Karnataka, India, Phone: +91 9611217065, e-mail: rekhapbadiger@gmail.com

How to cite this article: Raghavendrachar RB, Bhat RA, Dhakaria V. Surgical and Survival Outcomes with Neoadjuvant Chemotherapy in Advanced Epithelial Ovarian Cancer: A Longitudinal Study in a Tertiary Cancer Center. J South Asian Feder Obst Gynae 2020;12(1):27-30.

Source of support: Nil

Conflict of interest: None

intervention thereby denying the patient of an opportunity to undergo surgery. Most of the studies have proven that no residual tumor at the end of the surgery is the most important independent variable predicting the overall survival.

The present study was taken up to analyze outcomes of IDS in terms of achieving no macroscopic disease at the end of surgery and outcomes of patients in terms of postoperative morbidity, overall survival (OS), and progression-free survival (PFS).

\section{AıM}

To evaluate the role of NACT followed by interval debulking and adjuvant chemotherapy on surgical and survival outcomes in advanced epithelial ovarian cancer (AEOCs). 


\section{Materials and Methods}

This was a retrospective and longitudinal study conducted at HCG Enterprises, Bengaluru, and included patients diagnosed with advanced epithelial ovarian/fallopian tube/primary peritoneal cancer from the period of January 2013 to April 2017.

\section{Inclusion Criteria}

- Biopsy/cytology proven stage III C/IV epithelial carcinoma of the ovary/fallopian tube/primary peritoneal cancer.

- Advanced disease involving the subdiaphragmatic area, liver surface, mesenteric involvement, and extensive peritoneal disease documented on CECT/PET-CT imaging.

- Patients undergoing NACT (paclitaxel and carboplatin) based on above inclusion criteria, followed by IDS and adjuvant chemotherapy

\section{Exclusion Criteria}

- Patients who received chemotherapy agents other than paclitaxel and carboplatin during NACT.

Patients suspected for ovarian/fallopian tube/primary peritoneal cancer were evaluated by thorough history and physical examination, followed by imaging with PET-CT/CECT and tumor marker CA-125. If imaging was suggestive of $A E O C$, i.e., gross ascites, omental mass, extensive upper abdominal disease-like subdiaphragmatic deposits, surface hepatic disease or disease above diaphragm in the form of supra-mammary lymph nodes, and malignant pleural effusion, then the diagnosis would be confirmed by ascitic/pleural fluid cytology, omental biopsy, or biopsy of the ovarian mass. In our institution, all patients with AEOC undergo NACT followed by IDS and adjuvant chemotherapy. All the study patients received NACT with paclitaxel $175 \mathrm{mg} / \mathrm{m}^{2}$ and carboplatin (AUC 5), once every 3 weeks, for 3 cycles. After NACT, the patients were reevaluated with CECT abdomen and pelvis/PET-CT and tumor marker CA-125, and the response to NACT was assessed. All the patients underwent IDS after completing NACT. The standard operative procedure that is cytoreductive surgery (CRS) included total abdominal hysterectomy with bilateral salpingo-oophorectomy + supra-colic omentectomy \pm pelvic lymph-node dissection \pm para-aortic lymph-node dissection, \pm peritonectomy, \pm bowel resection. The type of cytoreductive surgery achieved was documented as complete cytoreduction when there was no residual disease at the end of surgery, optimal cytoreduction when $<1 \mathrm{~cm}$ residual disease was left behind, and suboptimal cytoreduction when $\geq 1 \mathrm{~cm}$ residual disease was left behind at the end of end of surgery. Perioperative complications if any were noted and graded on a scale of 0-5 according to Memorial Sloan Kettering Cancer Center surgical secondary events grading system. ${ }^{5}$ Adjuvant chemotherapy agents were paclitaxel $175 \mathrm{mg} / \mathrm{m}^{2}$ and carboplatin (AUC 5), once every 3 weeks, for 3 cycles.

The tumor histology and grade was obtained from pathologist and documented.

After completing treatment, patients were followed up every 3 months with clinical examination, CA-125, and imaging study like USG, CECT, or PET-CT scan. Disease recurrence was confirmed by clinical examination followed by CECT/PET-CT of abdomen and pelvis and CA-125 elevation and documented. The patients were followed up till December 2017. Minimum follow-up period was 5 months from last treatment and maximum follow-up period was
24 months from last treatment. The median follow-up period was 14.5 months.

\section{Statistical Analysis}

- Survival analysis was done using log-rank analysis.

- Differences in overall survival (OS) and progression-free survival (PFS) were analyzed using log-rank test (Log-rank Mantel-Cox test) with 24 months as the maximum follow-up time since most of the patients recur within this time.

- In inferential statistics, Chi-square test and Fisher's exact test were used to find out association between categorical variables.

- Differences in proportions were calculated using Chi-square analysis.

- SPSS version 23 was used to analyze the data and to obtain results.

- $p$ value $<0.05$ was considered as statistically significant.

\section{ResULts}

A total of 50 patients satisfied the inclusion criteria during the study period from January 2013 to April 2017. We found that majority of our patients were above 45 years of age (88\%) and were of postmenopausal status ( $80 \%)$. The most common presenting symptom was abdominal distension ( $56 \%)$, followed by abdominal pain (32\%) and other symptoms (12\%). Hypertension was seen in $30 \%$ of the patients and diabetes mellitus affected $26 \%$ of the patients in this study. Forty-three (86\%) patients of the study group had stage III C disease and 28 patients (14\%) had stage IV disease.

On histopathological evaluation, 47 (94\%) patients had serous tumor, mucinous, and endometrioid, and clear cell tumors were seen in one patient each.

At surgery, total abdominal hysterectomy with bilateral salpingo-oophorectomy with total omentectomy was performed. Also, 19 patients underwent retroperitoneal lymphadenectomy, 6 patients underwent peritonectomies ( 2 patients underwent pelvic peritonectomy and 4 patients underwent total peritonectomy), and one patient required anterior resection and another patient underwent right hemicolectomy with anterior resection and ileostomy.

There were no postoperative complications in 39 (78\%) patients. Grade I postoperative complication was seen in 8 (16\%), grade II, IV and V complications were seen in one patient each ( $2 \%$ each). Grade I postoperative complication was noted in the form of superficial wound gape, which required outpatient wound dressing resuturing under local anesthesia. Grade II complication was seen in one patient who developed wound infection requiring admission to hospital, intravenous antibiotic, and vacuum dressing followed by resuturing under local anesthesia. Both the patients who required resuturing had undergone bowel resection during IDS. A grade IV complication was noted in a patient who developed burst abdomen during adjuvant chemotherapy and required relaparotomy. A grade $\mathrm{V}$ complication was seen in one patient who developed postoperative sepsis and multiorgan failure leading to death of the patient. On analyzing the response to chemotherapy by RECIST criteria (response evaluation criteria in solid tumors), it was found that 39 patients had partial response, one patient had complete response, 5 patients had stable disease, and 5 patients has progressive disease. ${ }^{6}$

Adjuvant chemotherapy was started from third week after surgery for those patients who recovered without any complications. Those patients who required wound resuturing 
had a delayed start by another week. Three cycles of paclitaxel and carboplatin were administered as adjuvant chemotherapy. One patient who underwent anterior resection and another patient with suboptimal debulking received liposomal doxorubicin and carboplatin in adjuvant chemotherapy cycles. The other patients with suboptimal debulking continued paclitaxel and carboplatin and then switched to oral etoposide/palliation measures in view of progressive disease.

On analyzing the outcome of surgery, out of the total 50 patients who underwent interval bulking surgery, 39 patients had complete cytoreduction, 4 patients had optimal cytoreduction (up to $1 \mathrm{~cm}$ of residual disease), and 7 patients had suboptimal cytoreduction ( $>1 \mathrm{~cm}$ residual disease) (Table 1 ).

On survival analysis for 24 months, the overall survival for the study group was $21.234 \pm 0.738$ months with $95 \% \mathrm{Cl}$, and progression-free survival was $20.324 \pm 0.974$ months.

Log-rank test was done to compare the three groups of patients. ${ }^{7}$ On comparison of patients who had complete debulking, optimal debulking, and suboptimal debulking, the overall survival was $22.152 \pm$ $0.782,22.207 \pm 1.464$, and $14.094 \pm 1.374$ months, respectively, with $95 \% \mathrm{Cl}, p=0.001$, which was statistically significant (Fig. 1).

Similarly on comparing the progression-free survival of patients who had complete debulking, optimal debulking, and suboptimal debulking, it was $21.473 \pm 0.950,20.530 \pm 2.833$, and $10.339 \pm 0.986$ months, respectively, with $95 \% \mathrm{Cl}, p=0.002$, which was statistically significant (Table 2 and Fig. 2).

On cox regression analysis, the overall survival after complete debulking was significantly higher than after suboptimal debulking ( $p=0.007)$; however, there was no statistically significant difference in OS between optimal debulking and suboptimal debulking $(p=0.328)$.

Also on cox regression analysis, the progression-free survival after complete debulking was significantly higher than after suboptimal debulking ( $p=0.011)$.

However, there was no statistically significant difference in PFS between optimal debulking and suboptimal debulking ( $p=0.515)$.

We further compared the stage of disease and survival outcomes and the results were as follows:

The overall survival of patients with stage IIIC and stage IV diseases was $21.719 \pm 0.713$ and $18.737 \pm 3.165$ months, respectively, with $95 \% \mathrm{Cl}, p=0.371$, which was not statistically significant.

The progression-free survival of patients with stage III and stage IV diseases was $20.716 \pm 0.963$ and $18.737 \pm 3.165$ months, respectively, with $95 \% \mathrm{Cl}, p=0.308$, which was not statistically significant.

\section{Discussion}

The management of ovarian cancer has long been primary debulking followed by adjuvant chemotherapy. But several randomized trial and meta-analyses have now shown that NACT followed by IDS and adjuvant chemotherapy gives similar survival outcomes with minimal perioperative complications and better quality of life. The NCCN guideline 2019 for ovarian cancers gives an option of NACT followed by IDS for patients who have a lesser likelihood of optimal cytoreduction at PDS. ${ }^{8}$

In the present study, we found that complete debulking is possible in a higher percentage of cases after NACT in advanced EOC, which was $78 \%$ in this study group. This reflected in survival outcomes where we found that the OS and PFS were higher in the patient group after complete debulking and it was statistically significant. We did not find any statistically significant difference in OS and PFS on comparing stage of disease.

In a study by Deo et al., after NACT, optimal debulking was achieved in $72 \%$ of the cases, which was similar to the present study where complete debulking was achieved in $78 \%$ of the cases. ${ }^{9}$ In the study by Deo, the OS and DFS after NACT followed by IDS and adjuvant chemotherapy were 32 and 31 months, respectively, at the end of 34 months follow-up. They found that the degree of optimal cytoreduction was the only factor affecting survival $(p<0.05)$. In the present study, the OS and PFS after similar management were 21 and 20 months, respectively.

In a study by Sonia Batra et al., optimal cytoreduction was achieved in $72 \%$ of the patients who underwent NACT followed by IDS, which was comparable to $78 \%$ complete cytoreduction rate in the present study. ${ }^{10}$

In the EORTC trial (Table 2) by Vergote et al., the OS and PFS survival after NACT and IDS with adjuvant chemotherapy were 30 months and 12 months, respectively, and in the CHORUS trial, the values were 24.5 months and 11.7 months, respectively. ${ }^{11}$ In study by Baruah et al., the OS and PFS after NACT followed by IDS and adjuvant chemotherapy were 26 months and 18 months,

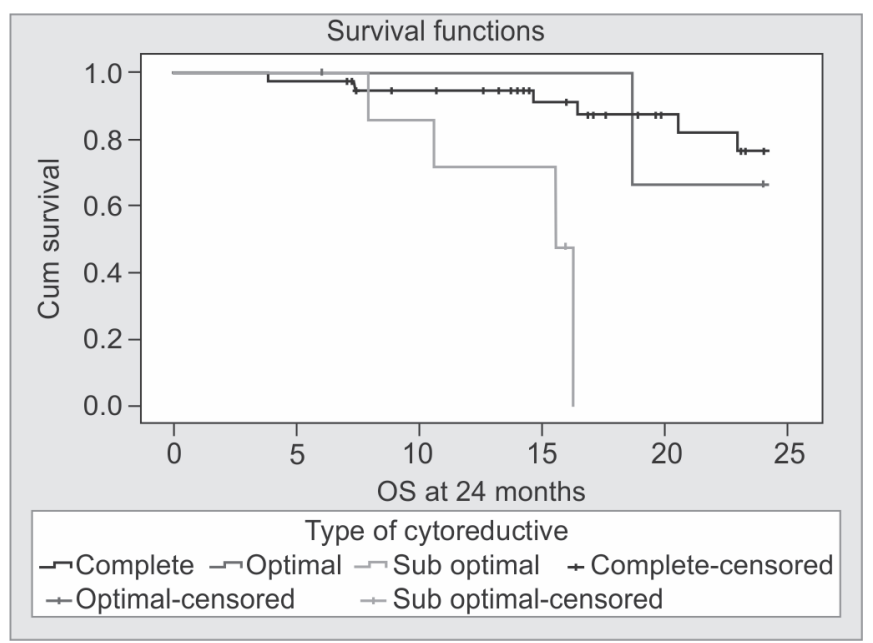

Fig. 1: Log-rank analysis of OS, $p=0.001$

Table 1: Log-rank analysis of OS and PFS in the study group

\begin{tabular}{lccclll}
\hline & \multicolumn{3}{c}{ Surgical outcome } & & \multicolumn{2}{c}{ Survival outcome } \\
\cline { 2 - 3 } \cline { 5 - 6 } \cline { 5 - 6 } & Number & Percentage & & OS & PFS \\
\hline Complete debulking & 39 & 78 & & $22.152 \pm 0.782$ & $21.473 \pm 0.950$ \\
Optimal debulking & 4 & 8 & & $22.207 \pm 1.464$ & $20.530 \pm 2.833$ \\
Suboptimal debulking & 7 & 14 & & $14.094 \pm 1.374$ & $10.339 \pm 0.986$ \\
Total & 50 & & & $p$ value 0.001 & $p$ value 0.002 \\
\hline
\end{tabular}


Table 2: Comparison of primary debulking surgery (PDS) and interval debulking surgery (IDS) results in other studies

\begin{tabular}{lccllll}
\hline Group & PDS & IDS & \multicolumn{2}{c}{ PFS } & \multicolumn{2}{c}{ OS } \\
\hline Vergote et al. & 336 & 334 & PDS-12 & IDS-12 & PDS-29 & IDS-30 \\
CHORUS trial & 276 & 274 & PDS-10.3 & IDS-11.7 & PDS-22.8 & IDS-24.5 \\
Baruah et al. & - & 104 & - & 18 & - & 26 \\
Georgeena et al. & 50 & 78 & 56 & 39 & 58 & 44 \\
Present study & - & 50 & - & 20.324 & - & 21.234 \\
\hline
\end{tabular}

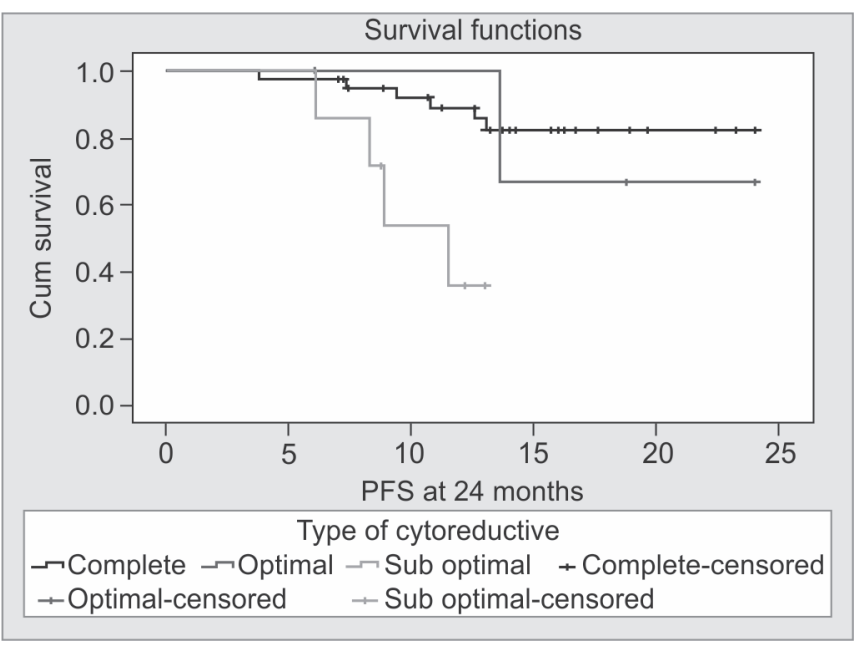

Fig. 2: Log-rank analysis of PFS, $p=0.002$

respectively, and in the present study, the OS and PFS after similar management were 21 and 20 months, respectively (Table 2$)^{12}$ In a study by Georgeena et al. involving 50 patients in PDS group and 78 patients in IDS group, the OS and PFS after PDS were 58 months and 56 months, respectively, and the OS and PFS after NACT were 44 months and 39 months, respectively, which were statistically significant differences. ${ }^{13}$ But in their study, the selection of cases for PDS or NACT followed by IDS was based on operability to achieve no residual disease and physical status of patient to withstand extensive surgery. Hence, the two study groups were not uniform (Table 2).

In the present study, the narrow difference between the OS and PFS could be due to the small numbers $(n=50)$ and also some of the patients in the study were recent recruits at the time of study. However, the OS and PFS were significantly longer for the patients who underwent complete debulking than after suboptimal debulking.

\section{Conclusion}

In advanced EOCs, NACT followed by IDS and adjuvant chemotherapy gives a better rate of complete debulking with minimal perioperative complications, thereby improving the prognosis for the patients. We conclude that the survival outcomes are better after NACT and IDS since the OS and PFS were significantly higher in our patients. The weakness of this study is the smaller number of patients and shorter duration of follow-up and also there was no group of patients who underwent primary debulking surgery to compare the outcomes. However, it can still be said that NACT followed by IDS in advanced EOC gives a better rate of complete cytoreduction with acceptable postoperative complications, thereby better survival outcomes.

\section{References}

1. Bray F, Ferlay J, Soerjomataram I, et al. Global cancer statistics 2018: GLOBOCAN estimates of incidence and mortality worldwide for 36 cancers in 185 countries. CA Cancer J Clin 2018;68(6):394-424. DOI: 10.3322/caac. 21492 .

2. National Cancer Registry Program. Incidence, Distribution, Trends in Incidence Rates and Projections of Burden of Cancer. March 2016. pp. 43-62.

3. du Bois A, Reuss A, Pujade-Lauraine E, et al. Role of surgical outcome as prognostic factor in advanced epithelial ovarian cancer: $A$ combined exploratory analysis of 3 prospectively randomized phase 3 multicenter trials. Cancer 2009;115(6):1234-1244. DOI: 10.1002/ cncr.24149.

4. Kehoe S, Hook J, Nankivell M, et al. Primary chemotherapy versus primary surgery for newly diagnosed advanced ovarian cancer (CHORUS): an open-label, randomised, controlled, noninferiority trial. Lancet 2015;386(9990):249-257. DOI: 10.1016/S01406736(14)62223-6.

5. Strong VE, Selby LV, Sovel M, et al. Development and assessment of memorial sloan kettering cancer center's surgical secondary events grading system. Ann Surg Oncol 2015;22(4):1061-1067. DOI: 10.1245/ s10434-014-4141-4.

6. Eisenhauer EA, Therasse P, Bogaerts J, et al. New response evaluation criteria in solid tumours: revised RECIST guideline (Version 1.1). Eur J Cancer 2009;45(2):228-247. DOI: 10.1016/j.ejca.2008.10.026.

7. Bland JM, Altman DG. The logrank test. BMJ 2004;328(7447):1073. DOI: 10.1136/bmj.328.7447.1073.

8. National Comprehensive Cancer Network. NCCN Clinical Practice Guidelines in Oncology (NCCN Guidelines ${ }^{\circledast}$ ). Ovarian cancer including fallopian tube cancer and primary peritoneal cancer. Version 2.2019. https://www.nccn.org/professionals/physician_gls/pdf/ovarian.pdf. Accessed September 17, 2019.

9. Deo SVS, Hemant G, Shukla NK, et al. Neoadjuvant chemotherapy followed by surgical cytoreduction in advanced epithelial ovarian cancer. Indian J Cancer 2006;43(3):117-121. DOI: 10.4103/0019509x.27933.

10. Sonia B, Himanshu N, Dave Kalpna, et al. Role of neoadjuvant chemotherapy (NACT) followed by surgical cytoreduction in advanced epithelial ovarian cancer. The J Obstet Gynecol India 2012;62(5):541-545. DOI: 10.1007/s13224-011-0106-8.

11. Vergote I, Tropé CG, Amant F, et al. Neoadjuvant chemotherapy or primary surgery in stage IIIC or IV ovarian cancer. N Engl J Med 2010;363(10):943-953. DOI: 10.1056/NEJMoa0908806.

12. Baruah U, Barmon D, Kataki A, et al. Neoadjuvant chemotherapy in advanced epithelial ovarian cancer: A survival study. Indian J Med Paediat Oncol 2015;36(1):38-42. DOI: 10.4103/0971-5851. 151781.

13. Georgeena P, Rajanbabu A, Vijaykumar D, et al. Surgical treatment pattern and outcomes in epithelial ovarian cancer patients from a cancer institute in Kerala, India. Ecancermedicalscience 2016;10:619. DOI: 10.3332/ecancer.2016.619. 